\title{
Länderübergreifende Zusammenarbeit in der Notfallmedizin
}

Ulrich Bürgi ${ }^{a}$,

Michael Christ ${ }^{b}$,

Barbara Hoganc,

Wilhelm Behringer ${ }^{\text {, }}$

Christoph Dodt ${ }^{e}$

a Präsident Schweizerische Gesellschaft für Notfall- und Rettungsmedizin (SGNOR), FAT Anästhesie und Intensivmedizin, FA Klinische Notfallmedizin, FA Notarzt, Aarau

b Zweiter Vorsitzender Deutsche Gesellschaft für Interdisziplinäre Notfall- und Akutmedizin (DGINA), Nürnberg

c Präsidentin European Society of Emergency Medecine (EuSEM), Hamburg

d Präsident Austrian Association of Emergency Medicine (AAEM), Wien

e Vorsitzender Deutsche Gesellschaft für Interdisziplinäre Notfall- und Akutmedizin (DGINA), München
Korrespondenz:

Dr. med. Ulrich Bürgi

Bereichsleiter Interdisziplinäres

Notfallzentrum Kantonsspital

CH-5001 Aarau

Tel. 0628385428

ulrich.buergi[at]ksa.ch

\section{Einleitung}

Im Jahre 2012 sind in den schweizerischen Notfallstationen schätzungsweise 1,5 Millionen Patienten behandelt worden. Seit rund 20 Jahren steigt die Anzahl der Notfallaufnahmen in der Schweiz kontinuierlich an und sie wird in den kommenden Jahren weiter ansteigen. Die Ursachen sind multifaktoriell und nach Regionen und Spitalauftrag unterschiedlich: demographische Veränderungen (Zunahme, Alterspyramide, Anteil fremder Ethnien), Rückgang der Grundversorgung (insbesondere in den städtischen Agglomerationen), höheres Gesundheitsbewusstsein der Bevölkerung und erleichterter Zugang zu Gesundheitsleistungen in Permanencen und Notfallpraxen zu Randzeiten. Die schweizerischen Notfallstationen sind durch die dauernde $\mathrm{Zu}$ nahme der Patienteneintritte belastet und erbringen die notwendigen Leistungen rund um die Uhr unter grossen personellen Anstrengungen.

Die Schweizerische Gesellschaft für Notfall- und Rettungsmedizin (SGNOR) setzt sich seit über $20 \mathrm{Jah}$ ren für fachliche und strukturelle Verbesserungen in den schweizerischen Notfallstationen ein. Die Probleme in der Schweiz gleichen denjenigen in Deutschland und Österreich. Die SGNOR hat sich deshalb entschieden, mit der Deutschen Gesellschaft für Interdisziplinäre Notfall- und Akutmedizin (DGINA) und der Austrian Association of Emergency Medicine (AAEM) enger zusammenzuarbeiten, um fachliche und strukturelle Massnahmen in gemeinsamen Konzepten und Programmen zu erarbeiten.

\section{Fünf Thesen zur Weiterentwicklung der Notfallmedizin in Deutschland, Österreich und der Schweiz}

\section{Ausgangslage}

Die rund um die Uhr stattfindende Notfallversorgung von Patienten mit akuten Gesundheitsstörungen ist eine unverzichtbare medizinische Dienstleistung für die Bevölkerung. In den letzten Jahren ist die Anzahl der Patienten, die eine notfallmedizinische Versorgung in einem Krankenhaus in Anspruch genommen haben, massiv gestiegen: Aktuell werden jährlich in Deutschland, Österreich und der Schweiz rund 25 Millionen Menschen (20\% der Bevölkerung) in den Krankenhäusern notfallmedizinisch versorgt. Betroffene Patienten haben Anspruch auf eine zeitnahe, risikoadaptierte und qualitativ hochwertige

\section{Zusammenfassung}

Die jährlich steigenden Patientenzahlen in den schweizerischen Notfallstationen belasten deren Mitarbeitende und die Infrastruktur der Spitäler zunehmend. Die Schweizerische Gesellschaft für Notfall- und Rettungsmedizin (SGNOR) setzt sich seit über 20 Jahren für fachliche und strukturelle Verbesserungen in der Notfall- und Rettungsmedizin ein. Da sich die Probleme in den Nachbarländern Deutschland und Österreich gleichen, haben die notfallmedizinischen Fachgesellschaften dieser 3 Länder, DGINA, AAEM und SGNOR, entschieden, enger zusammenzuarbeiten. Sie stellen in diesem Artikel 5 Thesen zur Weiterentwicklung der Notfallmedizin vor. Gleichzeitig werden die Definitionen für die Begriffe «medizinischer Notfall» und «Notfallmedizin» präzisiert.

Versorgung der als Bedrohung empfundenen Gesundheitsstörungen. Die gesundheitspolitischen und ökonomischen Rahmenbedingungen erfordern zusätzlich eine effiziente und effektive notfallmedizinische Versorgung.

\section{«Die Probleme in der Schweiz gleichen denjenigen in Deutsch- land und Österreich.»}

Der soziodemographische Wandel führt bei vielen Notfallpatienten zu einer Zunahme von Multimorbidität und medizinischer Komplexität, die eine besondere fachübergreifende Herausforderung darstellt, der durch die hochspezialisierte Medizin und ihre zunehmende Segmentierung und Aufgabenteilung nicht begegnet werden kann. Diese Herausforderungen an ein symptombezogenes und ganzheitliches Vorgehen in Diagnostik und Therapie erfordern spezielle Strukturen und Organisationsformen sowie 


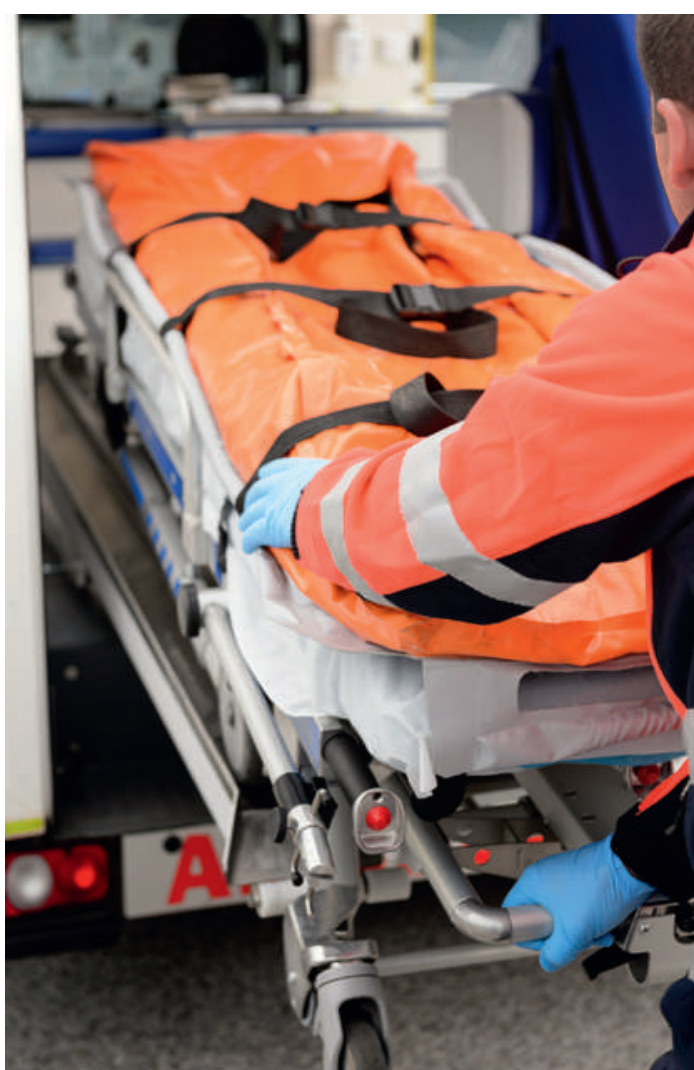

Die rund um die Uhr stattfindende Notfallversorgung ist eine unverzichtbare medizinische Dienstleistung.

eine spezifische, notfallmedizinische Ausbildung. In den meisten Ländern der Welt ist deshalb Notfallmedizin seit Jahren als eigenes Fachgebiet etabliert. Notfallmedizin ist eine faktische Realität.

\section{Die notfallmedizinischen Fachgesellschaften von Deutschland, Österreich und der Schweiz (DGINA, AAEM, SGNOR) veröffentlichen folgende 5 Thesen:}

1. Die Entwicklung der Notfallmedizin auf klinischer und struktureller Ebene sowie in der Fortund Weiterbildung als eigenständiges Fachgebiet mit eigenständigen Strukturen und Organisationsformen ist Voraussetzung für eine exzellente notfallmedizinische Versorgung.

2. Einführung des Facharztes/-ärztin für Notfallmedizin mit einer fünfjährigen Weiterbildung.

3. Empfehlung der Fachgesellschaften, dass in Deutschland, Österreich und der Schweiz möglichst bald Fachpflegende für Notfallmedizin in allen Notaufnahmen bzw. Notfallstationen Bestandteil des Pflegeteams sind.

4. AAEM, DGINA und SGNOR streben in den kommenden Jahren die Erfassung und Analyse der notfallmedizinischen Qualitätsparameter an.

5. Etablierung und Förderung der akademischen Forschung und Lehre für das Fach Notfallmedizin.

\section{Vorstand SGNOR}

PD Dr. med. Mathias Zürcher, Vizepräsident, Basel; Prof. Dr. François Sarasin, Vizepräsident, Genf; Prof. Dr. med. Roland Bingisser, Basel; Dr. med. Ünal Can, Zürich; Dr. med. Adam-Scott Feiner, Lausanne; Dr. med. Walter Hanhart, Neuchâtel; Gabriela Kaufmann, Bern; Prof. Dr. med. Dagmar Keller, Zürich; Dr. med. Beat Lehmann, Bern; Dr. med. Stefan Müller, Zürich; Dr. med. Peter Rupp, Spiez; Dr. med. Barbara Schild, Bellinzona; Dr. med. Robert Sieber, St. GalIen; Dr. med. Simon Sulser, Winterthur.

Durch Umsetzung dieser Thesen wird auch in Deutschland, Österreich und der Schweiz eine exzellente notfallmedizinische Versorgung trotz ökonomischen Einschränkungen nach internationalen Standards garantiert. Gleichzeitig wird dadurch das Policy Statement der «Union Européenne des Médecins Spécialistes» (UEMS) in der Fassung vom 20. April 2013 auch in deutschsprachigen Ländern umgesetzt (www.dgina.de/media/nachricht/2013_policy_ statement_UEMS.pdf).

\section{Definitionen}

\section{Medizinischer Notfall}

Als medizinischer Notfall bzw. als Notfallpatienten werden alle Personen definiert, die körperliche oder psychische Veränderungen im Gesundheitszustand aufweisen, für die der Patient selbst oder eine Drittperson unverzügliche medizinische und pflegerische Betreuung als notwendig erachtet.

\section{Das Fachgebiet Notfallmedizin}

Notfallmedizin ist eine Fachdisziplin, in der das Wissen und die praktischen Fähigkeiten zusammengefasst sind, um akute und dringliche Erkrankungen und Verletzungen von Personen zu verhindern bzw. diese $\mathrm{zu}$ diagnostizieren und $\mathrm{zu}$ behandeln. Die Fachdisziplin befasst sich mit einem umfassenden Spektrum physischer Störungen und Verhaltensauffälligkeiten von Notfallpatienten. Notfallmedizin definiert sich nicht durch den Ort des notfallmedizinischen Handelns, sondern durch die Inhalte der Fachlichkeit «Notfallmedizin» und umfasst daher präklinische und klinische Notfallsituationen.

In der Notfallmedizin ist die zeitkritische Versorgung von entscheidender Bedeutung. Die klinische Praxis der Notfallmedizin umfasst die präklinische und klinische Triage, die Erstevaluation, die akute Stabilisierung des betroffenen Patienten und das Management dringlicher und kritischer Fälle bis zur Entlassung in die ambulante Weiterversorgung oder die Überweisung zu einem anderen Arzt, ambulanten oder stationären Einrichtungen oder anderen Mitarbeitern des Gesundheitswesens. Das Fachgebiet umfasst auch die Teilnahme an der Weiterentwicklung der präklinischen Notfallsysteme und dem Massenanfall von Verletzten oder Erkrankten (MANV). 